\title{
Perfil de sensibilidade a antimicrobianos de bactérias isoladas do trato respiratório baixo de pacientes com pneumonia internados em hospitais brasileiros - Resultados do Programa SENTRY, 1997 e 1998
}

\author{
Hélio S. SAder ${ }^{1}$, Rodrigo E. Mendes ${ }^{1}$, Ana C. Gales ${ }^{1}$, Ronald N. JoneS ${ }^{2}$, \\ Michael A. PFAlleR ${ }^{2}$, CASSia ZOCCOLI ${ }^{3}$, JORGE SAMPAIO ${ }^{4}$
}

\begin{abstract}
Introdução: Pneumonia hospitalar é a mais fatal das infecções hospitalares, com taxas de mortalidade de 30 a $60 \%$. Estima-se que $15 \%$ de todas as mortes associadas à hospitalização estejam diretamente relacionadas a pneumonias hospitalares. O SENTRY é um estudo de vigilância de resistência a antimicrobianos envolvendo centros médicos em todo o mundo. Objetivo: Avaliar a sensibilidade a antimicrobianos de bactérias isoladas no trato respiratório baixo de pacientes com pneumonia internados em hospitais brasileiros. Material e métodos: Foram avaliadas 525 amostras bacterianas de 11 hospitais brasileiros, como parte do programa SENTRY. Os isolados foram testados por microdiluição em caldo contra um grande número de antimicrobianos. Resultados: As cinco espécies mais freqüentes foram (n/\%): Pseudomonas aeruginosa (158/30,1\%), Staphylococcus aureus (103/19,6\%), Acinetobacter spp. (68/13,0\%), Klebsiella spp. (50/9,5\%), e Enterobacter spp. (44/8,4\%). Essas cinco espécies representam mais de $80 \%$ de toda a amostragem. A $P$. aeruginosa apresentou altas taxas de resistência à maioria dos antimicrobianos testados. As maiores

taxas de sensibilidade foram apresentadas por piperacilina/tazobactam $(71,5 \%)$ e meropenem (69,0\%). Os compostos com maior atividade in vitro contra Acinetobacter spp. foram imipenem e meropenem (80,9\% de sensibilidade) seguido pela tetraciclina (63,2\%). A sensibilidade das amostras

de Klebsiella spp. foi muito baixa. MICs $\geq 2 \mu \mathrm{g} / \mathrm{mL}$ para ceftriaxona ou ceftazidima, indicando produção de ESBL, foram encontrados em $36,0 \%$ das amostras. Os antimicrobianos mais ativos contra

Klebsiella spp. foram os carbapenens (100\% de sensibilidade) e as quinolonas $(92,0 \%$ de sensibilidade). Ceftriaxona foi ativa contra somente $56,8 \%$ das amostras de Enterobacter spp. (MIC50,

$1 \mu \mathrm{g} / \mathrm{mL}$ ), enquanto a cefepima foi ativa contra 88,6\% destes isolados (MIC50, $\leq 0,12 \mu \mathrm{g} / \mathrm{mL}$ ). A resistência à oxacilina foi detectada em $43,7 \%$ dos isolados de $S$. aureus. As drogas mais ativas contra essa espécie foram: vancomicina, teicoplanina, quinupristin-dalfopristin e linezolida. Conclusões: Os resultados do presente estudo mostraram alta prevalência de Acinetobacter spp. e altas taxas de resistência entre bacilos gram-negativos quando comparados com resultados de estudos norte-
\end{abstract} americanos e europeus.(J Pneumol 2001;27(2):59-67)

\section{Antimicrobial susceptibility of bacteria isolated from the lower respiratory tract of inpatients with pneumonia in Brazilian hospitals - Results from the SENTRY surveillance program, 1997 and 1998}

\footnotetext{
Background: Nosocomial pneumonia is the most common fatal nosocomial infection with attributable mortality rates ranging from 30 to $60 \%$ and a rapid initiation of optimal
}

\footnotetext{
1. Laboratório Especial de Microbiologia Clínica, Disciplina de Doenças Infecciosas e Parasitárias da Universidade Federal de São Paulo, São Paulo.

2. Departmento de Patologia da Faculdade de Medicina da Universidade de Iowa, Iowa City, Iowa, EUA.

3. Laboratório Santa Luzia, Florianópolis, SC.

4. Laboratório Lâmina, Rio de Janeiro, RJ.
}

Endereço para correspondência - Dr. Helio S. Sader, Laboratório Especial de Microbiologia Clínica, Disciplina de Doenças Infecciosas e Parasitárias, Universidade Federal de São Paulo-EPM, Rua Botucatu, 740 - 04023-900 - São Paulo, SP. Tels.: (11) 5081-2819/5571-5180/ 5576-4393; Fax: (11) 5543-3013; E-mail: heliosader@uol.com.br

Recebido para publicação em 11/5/00. Reapresentado em 20/ 9/00. Aprovado, após revisão, em 16/10/00. 
antimicrobial therapy is important to obtain treatment success. SENTRY is a comprehensive antimicrobial surveillance study involving a great number of medical centers distributed worldwide. Objective: To evaluate the antimicrobial susceptibility of bacterial isolates collected from the lower respiratory tract of inpatients with pneumonia. Material \& methods: The authors report the antimicrobial susceptibility of 525 isolates collected in 11 Brazilian hospitals, as part of the SENTRY program. The isolates were tested for susceptibility by broth micro-dilution against a large number of drugs. Results: The five most frequently isolated species were (n/\%): Pseudomonas aeruginosa (158/30.1\%), Staphylococcus aureus (103/19.6\%), Acinetobacter spp. (68/ 13.0\%), Klebsiella spp. (50/9.5\%), and Enterobacter spp. (44/8.4\%). These five species represented more than $80 \%$ of all isolates. P. aeruginosa demonstrated high rates of resistance to most antimicrobial agents tested. The highest susceptibility rates were shown by piperacillin/ tazobactam (71.5\%) and meropenem (69.0\%). Acinetobacter spp. also showed very high rates of resistance. The most active compounds against this species were imipenem and meropenem

(80.9\% susceptibility) followed by tetracycline (63.2\% susceptibility). Cephalosporin susceptibilities among Klebsiella spp were very low and $36.0 \%$ of isolates were considered ESBL producers based on increased MICs, $\geq 2 \mu \mathrm{g} / \mathrm{mL}$ ) to ceftriaxone or ceftazidime or aztreonam. Ceftriaxone was active against only $56.8 \%$ of Enterobacter spp. isolates (MIC50 $1 \mu \mathrm{g} / \mathrm{mL}$ ), while cefepime was active against $88.6 \%$ of these isolates (MIC, $\leq 0.12 \mathrm{mg} / \mathrm{mL}$ ). Oxacillin-resistance was detected in $43.7 \%$ of $\mathrm{S}$. aureus isolates. The most active drugs against this species were vancomycin, teicoplanin, quinupristin/dalfopristin, and linezolid. Conclusion: The results of this study demonstrated a higher prevalence of Acinetobacter spp. and higher resistance rates among Gram-negative rods when compared with results from North American and European studies.

Descritores - Testes de sensibilidade microbiana. Resistência microbiana a drogas. Análise bacteriana. Pneumonia bacteriana. Infecção hospitalar.

Key words - Microbial sensitivity tests. Microbial drug resistance. Bacterial analysis. Bacterial pneumonia. Nosocomial infection.

\section{INTRODUÇÃO}

Em unidades de terapia intensiva (UTIs), a pneumonia é a infecção mais freqüente na Europa ${ }^{(1,2)}$ e a segunda infecção mais freqüente nos hospitais americanos( ${ }^{(3)}$. Ela acontece em 0,6 a $2 \%$ dos pacientes hospitalizados e está relacionada a altas taxas de mortalidade atribuída (30 a $60 \%)^{(4,5)}$. As altas taxas de mortalidade encontradas em pneumonias hospitalares refletem em grande parte fatores relacionados ao paciente, à instituição e a características regionais. Como muitos pacientes com pneumonia hospitalar apresentam também outras patologias de base, imunossupressão, alteração do nível de consciência e/ou disfunção cardiopulmonar, o início rápido da terapia antimicrobiana adequada é essencial para obter sucesso terapêutico(3,4,6).

A resistência a antimicrobianos tem aumentado drasticamente nos últimos anos ${ }^{(7,8)}$. Conseqüentemente, a abordagem do paciente com pneumonia hospitalar também tem sofrido alterações e uma série de fatores devem ser levados em consideração antes do início da terapêutica
Siglas e abreviaturas utilizadas neste trabalho

ATCC - American type culture collection

CDC - Centers for Disease Control and Prevention

ESBL - Extended spectrum $\beta$-lactamases

FDA - Food and Drugs Administration

MIC - Concentração inibitória mínima

NCCLS - National Committee for Clinical Laboratory Standards ORSA - S. aureus resistente à oxacilina

UTI - Unidade de terapia intensiva

CESP - Citrobacter, Enterobacter, Serratia e Providencia

antimicrobiana empírica. A escolha da terapêutica empírica é extremamente importante, pois uma evolução clínica desfavorável poderá ocorrer se a terapêutica antimicrobiana adequada for retardada. Vários critérios podem ser utilizados para facilitar a escolha da terapêutica antimicrobiana empírica ${ }^{(5)}$. O reconhecimento dos patógenos mais prováveis parece ser um dos mais importantes fatores $^{(9)}$. Porém, os resultados do perfil de sensibilidade a antimicrobianos desses patógenos devem ser obtidos através de estudos de vigilância locais e regionais e considerados na escolha da terapêutica antimicrobiana empíri$\mathrm{ca}^{(5,7,8)}$.

O "SENTRY Antimicrobial Surveillance Program (SENTRY)" é um programa mundial e longitudinal de vigilância de resistência a antimicrobianos. Esse programa apresenta as seguintes características principais: a) avalia várias regiões do mundo de forma longitudinal; b) utiliza testes de 
sensibilidade de referência para avaliação das bactérias; c) coleta bactérias dos importantes sítios de infecções; d) avalia um grande número de antimicrobianos; e) utiliza técnicas moleculares de tipagem para avaliação do modo de disseminação de cepas multirresistentes; f) avalia os mecanismos de resistência em nivel molecular; g) divulga rapidamente os resultados em congressos e revistas médicas ${ }^{(10)}$. A coleta de amostras é feita de acordo com o sítio de infecção e inclui cinco objetivos: a) infecções da corrente sanguínea; b) infecções comunitárias do trato respiratório; c) pneumonia hospitalar; d) infecção de ferida cirúrgica; e) infecções do trato urinário. No presente artigo analisamos 525 bactérias isoladas do trato respiratório inferior de pacientes com pneumonia hospitalar internados em hospitais brasileiros.

\section{Material e MÉTOdos}

Amostras bacterianas: As bactérias foram coletadas em 11 hospitais brasileiros através de três laboratórios de microbiologia distribuídos em três cidades: São Paulo, Rio de Janeiro e Florianópolis. Em São Paulo as amostras foram coletadas pelo Laboratório Especial de Microbiologia Clínica (Disciplina de Doenças Infecciosas e Parasitárias da Escola Paulista de Medicina-Unifesp) de pacientes internados no Hospital São Paulo. No Rio de Janeiro as bactérias foram coletadas pelo Laboratório Lâmina em cinco hospitais da cidade: Hospital Pró-Cardíaco, Hospital Samaritano, Clínica Banbina I e II, Centro de Prematuro do Rio de Janeiro e Hospital de Clínicas de Ipanema. Ao contrário de São Paulo, no Rio de Janeiro as amostras são coletadas em pequenos hospitais privados, mas que também apresentam sérios problemas de resistência bacteriana. Em Florianópolis as amostras foram coletadas pelo Laboratório Médico Santa Luzia também em vários hospitais da cidade, tais como Hospitais de Caridade, Instituto de Cardiologia, Hospital Regional Homero de Miranda, Maternidade Carmela Dutra e Hospital dos Servidores.

Cada laboratório participante enviou aproximadamente 100 amostras bacterianas coletadas consecutivamente entre julho e dezembro de 1997 e 1998. Todas as amostras foram coletadas de pacientes hospitalizados e somente uma amostra de cada paciente foi incluída no estudo. As bactérias avaliadas foram isoladas de escarros, secreções traqueais e lavados brônquicos ou broncoalveolares. Foram incluídas no estudo apenas bactérias isoladas de espécimes clínicos que preenchessem os critérios de aceitabilidade preconizados pela Sociedade Americana de Microbiologia, isto é, baixo número de células epiteliais e alto número de leucócitos, além de correlação com o resultado do exame direto de gram ${ }^{(11)}$. As bactérias foram identificadas (gênero e espécie) pelo laboratório partici- pante e enviadas para o centro coordenador (Universidade de Iowa, Iowa City, IA, EUA) para avaliação da sensibilidade a antimicrobianos e demais testes laboratoriais.

Avaliação da sensibilidade a antimicrobianos: Foi realizada pela técnica de microdiluição em caldo conforme descrito pelo National Committee for Clinical Laboratory Standard (NCCLS)(12), que é o órgão responsável pela padronização de técnicas laboratoriais nos EUA. Os antimicrobianos foram obtidos dos respectivos fabricantes e incluem a maioria dos antibióticos disponiveis comercialmente. Também foram testados antimicrobianos que ainda se encontram ou se encontravam em fase final de pesquisa no periodo do estudo, tais como a fluoroquinolona gatifloxacina (Tequin ${ }^{\circledR}$, Bristol Myers Squibb), a estreptogramina quinupristin/dalfopristin (Synercid ${ }^{\circledR}$, Rhone Poulanc Rorer) e a oxazolidinona linezolida (Pharmacia \& Upjohn).

Controle de qualidade: Todos os testes foram realizados sob rígido controle de qualidade através da avaliação das seguintes cepas controles: Escherichia coli ATCC 25922, Staphylococcus aureus ATCC 29213, Pseudomonas aeruginosa ATCC 27853, e Enterococcus faecalis ATCC 29212. A interpretação dos testes, isto é, a categorização das amostras em resistente, intermediária ou sensivel, foi realizada de acordo com a padronização recomendada pelo NCCLS ${ }^{(13)}$.

Avaliação das amostras produtoras de $\beta$-lactamases de espectro estendido (ESBL): Amostras de Escherichia coli e Klebsiella pneumoniae foram selecionadas através de screening preconizado pelo NCCLS ${ }^{(14)}$. Cepas com MICs elevados $(\geq 2 \mu \mathrm{g} / \mathrm{mL})$ para ceftazidima ou ceftriaxona ou aztreonam foram consideradas como possiveis produtoras de ESBL e confirmadas através da comparação da sensibilidade (MIC) aos quatro substratos $\beta$-lactâmicos (ceftazidima, cefuroxima, cefotaxima e ceftriaxona) isoladamente com a sensibilidade a esses substratos associados ao inibidor de $\beta$-lactamase ácido clavulânico (concentração fixa de $2 \mu \mathrm{g} / \mathrm{mL}$ ) utilizando fitas de Etest $^{\circledR}$ (AB Biodisk, Solna, Suécia) especialmente preparadas para essa finalidade. Uma amostra foi caracterizada como produtora de ESBL quando a associação com o ácido clavulânico levou a queda superior a duas diluições logarítmicas $\left(\log _{2}\right)$ em comparação com o MIC do mesmo substrato isolado ${ }^{(15)}$.

\section{RESULTADOS}

A distribuição das dez espécies mais freqüentes se encontra na Tabela 1. Essas dez espécies foram responsáveis por $95,5 \%$ dos casos avaliados. Os bacilos gram-negativos e os cocos gram-positivos foram isolados em $71,9 \%$ e $23,6 \%$ desses casos, respectivamente.

A Tabela 2 resume os resultados de sensibilidade a antimicrobianos, MIC50 e MIC90 dos cinco bacilos gram- 
TABELA 1

Classificação das espécies bacterianas de acordo com a prevalência encontrada para as amostras isoladas em espécimes respiratórios de pacientes com pneumonia hospitalar em comparação com o total de amostras brasileiras

\begin{tabular}{lrr}
\hline \multicolumn{1}{c}{ Ordem de prevalência } & \% & n \\
\hline Pseudomonas aeruginosa & 30,1 & 158 \\
Staphylococcus aureus & 19,6 & 103 \\
Acinetobacter spp. & 13,0 & 68 \\
Klebsiella spp. & 9,5 & 50 \\
Enterobacter spp. & 8,4 & 44 \\
Enterococcus spp. & 4,0 & 21 \\
Serratia spp. & 4,0 & 21 \\
Escherichia coli & 3,4 & 18 \\
Stenotrophomonas maltophilia & 2,5 & 13 \\
Proteus spp. & 1,0 & 5 \\
\hline
\end{tabular}

negativos mais freqüentemente isolados do trato respiratório inferior frente a 22 antimicrobianos de diversas classes, incluindo $\beta$-lactâmicos, aminoglicosídeos, fluoroquinolonas, macrolídeos, glicopeptídeos, tetraciclinas e sulfas.

A $P$. aeruginosa foi o patógeno mais freqüente (158 casos - 30,1\%) e demonstrou altas taxas de resistência para a maioria dos antibióticos testados. Os antibióticos mais ativos contra essa espécie foram a associação pipe-

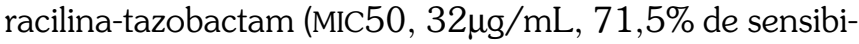
lidade) e o carbapenem meropenem (MIC50, $1 \mu \mathrm{g} / \mathrm{mL}$; $69,0 \%$ de sensibilidade). A fluoroquinolona ciprofloxacina também apresentou boa potência com baixos MICs (MIC50, 0,50 $\mu \mathrm{g} / \mathrm{mL}$ ); porém, a percentagem de bactérias sensiveis a esse antibiótico $(61,4 \%)$ foi um pouco inferior àquela apresentada pelos $\beta$-lactâmicos mais poten-

TABELA 2

Perfil de sensibilidade das bactérias gram-negativas mais freqüentemente isoladas do trato respiratório baixo de pacientes com infecção hospitalar em hospitais brasileiros em 1997 e 1998

\begin{tabular}{|c|c|c|c|c|c|c|c|c|c|c|}
\hline \multirow[t]{2}{*}{ Classe/antibiótico } & \multicolumn{2}{|c|}{$\begin{array}{l}\text { P. aeruginosa } \\
(1 / 158)\end{array}$} & \multicolumn{2}{|c|}{$\begin{array}{c}\text { Acinetobacter spp. } \\
(3 / 68)\end{array}$} & \multicolumn{2}{|c|}{$\begin{array}{c}\text { Klebsiella spp. } \\
(4 / 50)\end{array}$} & \multicolumn{2}{|c|}{$\begin{array}{c}\text { Enterobacter spp. } \\
(5 / 44)\end{array}$} & \multicolumn{2}{|c|}{$\begin{array}{l}\text { Serratia spp. } \\
\quad(6 / 21)\end{array}$} \\
\hline & $\mathrm{MIC} 50 / 90$ & $\%$ Sens. & $\mathrm{MIC} 50 / 90$ & $\%$ Sens. & MIC50/90 & $\%$ Sens. & MIC50/90 & $\%$ Sens. & MIC50/90 & $\%$ Sens. \\
\hline \multicolumn{11}{|l|}{ Cefalosporinas } \\
\hline Cefazolina & $>16 />16$ & 0,0 & $>16 />16$ & 0,0 & $\leq 0,25 / 0,5$ & 60,0 & $>16 />16$ & 9,1 & $>16 />16$ & 0,0 \\
\hline Cefoxitina & $>32 />32$ & 0,0 & $>32 />32$ & 0,0 & $4 />32$ & 80,0 & $>32 />32$ & 11,4 & $>32 />32$ & 0,0 \\
\hline Ceftriaxona & $>32 />32$ & 5,1 & $>32 />32$ & 7,4 & $\leq 0,25 />32$ & $68,0(36,0)^{\mathrm{a}}$ & $1 />32$ & 56,8 & $8 />32$ & 66,7 \\
\hline Cefazidima & $8 />16$ & 57,6 & $>16 />16$ & 10,3 & $0,25 />16 \quad 7$ & $76,0(36,0)^{\mathrm{a}}$ & $0,5 />16$ & 59,1 & $1 />16$ & 71,4 \\
\hline Cefepima & $8 />16$ & 63,9 & $>16 />16$ & 20,6 & $\leq 0,12 />16$ & 86,0 & $\leq 0,12 / 16$ & 88,6 & $0,25 / 2$ & 100,0 \\
\hline \multicolumn{11}{|l|}{$\overline{\text { Outros - } \beta \text {-lactâmicos }}$} \\
\hline Ampicilina & $>16 />16$ & 0,0 & $>16 />16$ & 1,5 & $>16 />16$ & 0,0 & $>16 />16$ & 4,5 & $>16 />16$ & 0,0 \\
\hline Meropenem & $1 />8$ & 69,0 & $1 />8$ & 80,9 & $\leq 0,06 / \leq 0,06$ & 100,0 & $\leq 0,06 / 0,12$ & 100,0 & $\leq 0,06 / 0,12$ & 100,0 \\
\hline \multicolumn{11}{|l|}{ Aminoglicosídeos } \\
\hline Gentamicina & $4 />16$ & 56,3 & $>16 />16$ & 25,0 & $0,5 />16$ & 76,0 & $1 />16$ & 75,0 & $16 />16$ & 47,6 \\
\hline Amicacina & $4 />32$ & 63,9 & $>32 />32$ & 14,7 & $2 / 32$ & 82,0 & $4 />32$ & 84,1 & $4 />32$ & 71,4 \\
\hline Tobramicina & $1 />16$ & 59,5 & $>16 />16$ & 39,7 & $1 />16$ & 68,0 & $1 />16$ & 61,4 & $16 />16$ & 33,3 \\
\hline \multicolumn{11}{|l|}{ Fluoroquinolonas } \\
\hline Ciprofloxacina & $0,5 />2$ & 61,4 & $>2 />2$ & 20,6 & $0,03 / 1$ & 92,0 & $0,03 />2$ & 81,8 & $1 />2$ & 57,1 \\
\hline Gatifloxacina & $2 />4$ & 55,7 & $>4 />4$ & 27,9 & $0,06 / 1$ & 92,0 & $0,06 / 4$ & 86,4 & $1 / 4$ & 66,7 \\
\hline Levofloxacina & $2 />4$ & 58,2 & $>4 />4$ & 20,6 & $\leq 0,5 / 1$ & 92,0 & $\leq 0,5 />4$ & 86,4 & $1 / 4$ & 71,4 \\
\hline Trovafloxacina & $1 />4$ & 52,5 & $>4 />4$ & 33,8 & $0,06 / 0,5$ & 92,0 & $0,06 />4$ & 81,8 & $2 />4$ & 47,6 \\
\hline Grepafloxacina & $1 />2$ & 51,6 & $>2 />2$ & 22,9 & $\leq 0,25 / 0,5$ & 91,3 & $\leq 0,25 />2$ & 86,7 & $2 />2$ & 40,0 \\
\hline
\end{tabular}

a Percentagem de cepas produtoras de ESBL segundo criterio de screening do NCCLS 1998. 
tes. Os antibióticos mais ativos contra $P$. aeruginosa em ordem decrescente de percentagem de amostras sensíveis foram os seguintes (\% sensibilidade): piperacilina/tazobactam $(71,5)>$ meropenem $(69,0 \%)>$ imipenem $(66,5 \%)>$ cefepima $=$ amicacina $(63,9 \%)>$ ciprofloxacina $(61,4 \%)>$ tobramicina $(59,5 \%)$.

Em nosso estudo o Acinetobacter spp. foi o terceiro patógeno mais freqüente e os carbapenens foram ativos contra $80,9 \%$ das amostras (MIC50, $1 \mu \mathrm{g} / \mathrm{mL}$ para imipenem e meropenem; Tabela 2). O próximo antibiótico mais ativo foi a tobramicina, que inibiu somente $39,7 \%$ das amostras (MIC50, $>16 \mu \mathrm{g} / \mathrm{mL}$ ).

As amostras de Klebsiella spp. também apresentaram taxas muito altas de resistência (Tabela 2). O carbapenem

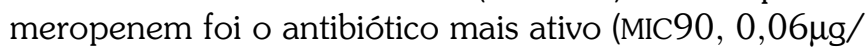
$\mathrm{mL}$ e $100 \%$ de sensibilidade), seguido pelo imipenem (MIC90, 0,5 $\mathrm{g} / \mathrm{mL}$ e $100 \%$ de sensibilidade). Cefepima apresentou a maior taxa de sensibilidade entre os outros $\beta$-lactâmicos avaliados. Ao utilizar os valores de corte estabelecidos pelo NCCLS ${ }^{(14)}$ para screening de amostras produtoras de $\beta$-lactamases de espectro estendido ou ampliado (extended-spectrum $\beta$-lactamases - ESBL), isto $e ́, \geq 2 \mu \mathrm{g} / \mathrm{mL}$ (o ponto de corte normal de sensibilidade é $8 \mu \mathrm{g} / \mathrm{mL}$ ) aproximadamente $36 \%$ das amostras de $K$. pneumoniae seriam consideradas produtoras de ESBL (Tabela 2). As amostras produtoras de ESBL apresentaram altas taxas de resistência cruzada com outras classes de antibióticos; porém, as quinolonas apresentaram excelente atividade contra essas amostras, inibindo mais de $90 \%$ do total de amostras de Klebsiella spp. avaliadas. A ciprofloxacina inibiu 92\% das amostras e apresentou MIC90 de $1 \mu \mathrm{g} / \mathrm{mL}$. As outras quinolonas avaliadas apresentaram desempenho muito semelhante (Tabela 2).

O Enterobacter spp. apresentou altas taxas de resistência às cefalosporinas de terceira geração, monobactans e penicilinas de amplo espectro associadas a inibidores de $\beta$-lactamases. Cerca de $40 \%$ das amostras testadas apresentaram resistência à ceftazidima, ceftriaxona, aztreonam e piperacilina/tazobactam (Tabela 2). Porém, a cefepima permanece bastante ativa contra Enterobacter spp. (88,6\% de sensibilidade) e outras espécies produtoras de $\beta$-lactamases cromossômicas induziveis (Classe $\mathrm{C}$ ou Grupo 1).

Cerca de metade das amostras de $S$. aureus foram resistentes à oxacilina $(56,3 \%$ de sensibilidade). A grande maioria das amostras de $S$. aureus resistentes à oxacilina (ORSA) apresentou resistência cruzada a outros antimicrobianos, tais como clindamicina (54,4\% de sensibilidade), ciprofloxacina (55,3\% de sensibilidade) e sulfametoxazol/ trimetoprim (58,3\% de sensibilidade). As novas quinolonas apresentaram atividade um pouco superior à oxacilina e ciprofloxacina. A gatifloxacina foi ativa contra $87,4 \%$ das amostras (MIC50, 0,12 $\mu \mathrm{g} / \mathrm{mL}$ e MIC $90,4 \mu \mathrm{g} / \mathrm{mL}$ ). Os
TABELA 3

Perfil de sensibilidade de amostras de $S$. aureus isoladas do trato respiratório baixo de pacientes com infecção hospitalar em hospitais brasileiros em 1997 e 1998

Espécie (ordem de prevalência $/ \mathrm{n}^{\circ}$ de bactérias testadas)

\begin{tabular}{|c|c|c|}
\hline \multirow[t]{2}{*}{ Classe/antibiótico } & \multicolumn{2}{|c|}{ S. aureus $(2 / 103)$} \\
\hline & MIC50/90 & $\%$ Susc. \\
\hline \multicolumn{3}{|l|}{ Cefalosporinas } \\
\hline Cefazolina & $\leq 2 />16$ & $56,3^{\mathrm{a}}$ \\
\hline Ceftriaxona & $4 />32$ & $56,3^{\mathrm{a}}$ \\
\hline Ceftazidima & $16 />16$ & 47,6 \\
\hline Cefepima & $4 />16$ & $56,3^{a}$ \\
\hline \multicolumn{3}{|l|}{ Outros $\beta$-lactâmicos } \\
\hline Oxacilina & $1 />8$ & $56,3^{\mathrm{a}}$ \\
\hline Penicilina & $32 />32$ & 7,8 \\
\hline Imipenem & $0,12 />8$ & $56,3^{\mathrm{a}}$ \\
\hline Amoxicilina/clavulanato & $2 />16$ & $56,3^{a}$ \\
\hline Piperacilina/tazobactam & $4 />64$ & $56,3^{a}$ \\
\hline \multicolumn{3}{|l|}{ MLS } \\
\hline Clindamicina & $0,25 />8$ & 54,4 \\
\hline Eritromicina & $>8 />8$ & 40,8 \\
\hline Doxiciclina & $1 />4$ & 83,5 \\
\hline \multicolumn{3}{|l|}{ Fluoroquinolonas } \\
\hline Ciprofloxacina & $0,5 />2$ & 55,3 \\
\hline Gatifloxacina & $0,12 / 4$ & 87,4 \\
\hline Trovafloxacina & $0,06 / 2$ & 88,3 \\
\hline Grepafloxacina & $\leq 0,25 />2$ & 61,9 \\
\hline \multicolumn{3}{|l|}{ Outros } \\
\hline Gentamicina & $0,5 />16$ & 56,3 \\
\hline Cloranfenicol & $16 />16$ & 48,5 \\
\hline Rifampicina & $\leq 0,25 />2$ & 63,1 \\
\hline Tetraciclina & $\leq 4 />8$ & 50,5 \\
\hline Trimetoprim/sulfametoxazol & $<0,5 />1$ & 58,3 \\
\hline Vancomicin & $1 / 1$ & 100,0 \\
\hline Teicoplanina & $1 / 2$ & 100,0 \\
\hline Quinupristin/dalfopristin & $0,5 / 1$ & 100,0 \\
\hline Linezilida & $2 / 4$ & 100,0 \\
\hline
\end{tabular}

a \% estabelecida através da suscetibilidade à oxacilina.

glicopeptídeos vancomicina (MIC50 e MIC90 de $1 \mu \mathrm{g} / \mathrm{mL}$ ) e teicoplanina (MIC50, $1 \mu \mathrm{g} / \mathrm{mL}$ e MIC $90,2 \mu \mathrm{g} / \mathrm{mL}$ ), a nova

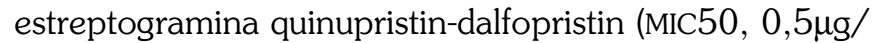
$\mathrm{mL}$ e MIC90, $1 \mu \mathrm{g} / \mathrm{mL}$ ) e a nova oxazolidinona linezolida (MIC50, $2 \mu \mathrm{g} / \mathrm{mL}$ e MIC90, $4 \mu \mathrm{g} / \mathrm{mL}$ ) foram os antibióticos mais ativos contra $S$. aureus, inibindo $100 \%$ das amostras avaliadas (Tabela 3).

\section{DISCUSSÃO}

A distribuição dos agentes que mais freqüentemente causam pneumonia hospitalar pode variar muito, de acordo com a região geográfica. A alta prevalência de bacilos 
gram-negativos não-fermentadores, especialmente Acinetobacter spp., como causa de pneumonia e outras infecções hospitalares, foi um dos resultados mais interessantes do Programa SENTRY no Brasil ${ }^{(16,17)}$. A importância desse patógeno no ambiente hospitalar aumentou rapidamente nos últimos anos ${ }^{(18)}$. A pressão seletiva causada pelo extensivo uso de antimicrobianos de amplo espectro é um dos principais fatores relacionados ao surgimento de colonização e infecção por esse patógeno ${ }^{(18)}$. Além disso, uma vez introduzido no ambiente hospitalar, sua erradicação se torna difícil $e$ a disseminação tanto intra quanto inter-hospitalar pode ocorrer de maneira rápida e ampla(19). Dessa maneira, a alta taxa de isolamento de Acinetobacter spp. neste estudo pode ser devida ao alto uso de antibióticos e/ou a falhas na implementação de medidas de controle de infecção hospitalar nas instituições avaliadas.

Pneumonias hospitalares são freqüentemente polimicrobianas, com predomínio de bactérias gram-negati$\operatorname{vas}^{(3,20)}$. A alta prevalência de bacilos gram-negativos nãofermentadores e enterobactérias encontrada nesse estudo sugere que a maioria das amostras se originaram de pacientes com longa internação e/ou uso prolongado de antibióticos $^{(21)}$. Esse fato explicaria também a baixa incidência de $H$. influenzae e $S$. pneumoniae, uma vez que a ocorrência de pneumonia hospitalar por esses patógenos está associada à ausência de uso prévio de antibióti$\cos ^{(22,23)}$. Porém, dificuldade na recuperação desses patógenos pelos centros participantes não pode ser completamente descartada.

Internação hospitalar prolongada, uso prévio de cefalosporinas de terceira geração e presença de doença pulmonar obstrutiva crônica são os principais fatores relacionados com pneumonia hospitalar por $P$. aeruginosa ${ }^{(24)}$. Essas pneumonias também estão relacionadas com taxas mais altas de mortalidade ${ }^{(25)}$. O mecanismo de resistência a carbapenens mais freqüentemente descrito em $P$. aeruginosa é decorrente da hiperprodução de $\beta$-lactamases cromossômicas induziveis (também conhecidas como Classe $\mathrm{C}$ ou $\mathrm{AmpC}$ ou Grupo 1) associada à diminuição de permeabilidade por perda de porinas $\mathrm{D} 2$ ou OprD. Como as cefalosporinas utilizam uma porina diferente para penetrar na bactéria, a perda desta porina D2 pode não afetar a sensibilidade às cefalosporinas. Dessa maneira, cepas de $P$. aeruginosa resistentes a carbapenens podem permanecer sensiveis às cefalosporinas de terceira (ceftazidima) ou quarta (cefepima) gerações ${ }^{(26)}$. As enzimas capazes de degradar carbapenens sem necessitar da ocorrência simultânea de perda de porinas, também chamadas de carbapenases, são raras nos Estados Unidos e na América Latina. Essas enzimas podem ser divididas em dois grupos: Classe A ou Grupo $2 \mathrm{f}$ de Bush-Jacoby-Medeiros e Classe B ou Grupo 3 de Bush-Jacoby-Medeiros ${ }^{(27)}$.
Estas últimas também são conhecidas como metaloenzimas e têm sido descritas principalmente no Japão. Tanto a perda de porina D2 quanto a produção de $\beta$-lactamases da Classe A ou B produziram maior resistência ao imipenem quando comparada com a resistência ao meropenem, validando assim a maior atividade do meropenem observada neste estudo (Tabela 2).

$O$ segundo patógeno mais freqüente foi o $S$. aureus (Tabelas 1 e 3). Cerca de metade das amostras analisadas apresentou resistência à oxacilina $e$, conseqüentemente, aos outros $\beta$-lactâmicos. Na verdade, as amostras de $S$. aureus resistentes à oxacilina (ORSA) apresentaram resistência cruzada também a antibióticos de outras classes, como eritromicina, clindamicina, cloranfenicol e sulfametoxazol/trimetoprim. Apesar de a grande maioria das amostras de ORSA terem apresentado resistência também à ciprofloxacina, as novas quinolonas foram ativas contra boa parte dessas amostras, indicando que essas drogas podem ser úteis no tratamento de infecções causadas por $S$. aureus resistentes à oxacilina e ciprofloxacina ${ }^{(28)}$. Das novas quinolonas avaliadas nesse estudo, somente a ciprofloxacina, a levofloxacina e a gatifloxacina se encontram disponíveis comercialmente no Brasil. A gatifloxacina pertence à nova geração de quinolonas que apresentam potência muito superior que as quinolonas anteriores contra bactérias gram-positivas, especialmente $S$. pneumoniae, e bactérias atípicas (micoplasma e clamídia), além de manter excelente atividade contra os bacilos gram-negativos hospitalares ${ }^{(28)}$. Dessa maneira, esse antibiótico deverá representar uma excelente opção terapêutica para o tratamento de pneumonias domiciliares e hospitalares.

A estreptogramina quinupristin/dalfopristin é um antibiótico com atividade contra cocos gram-positivos, incluindo $S$. aureus resistente à oxacilina e Enterococcus faecium resistente à vancomicina. Porém, não apresenta atividade contra $E$. faecalis e já têm sido relatado casos de E. faecium resistentes a esse antimicrobiano ${ }^{(29)}$. A linezilida ainda não se encontra disponível comercialmente, mas foi recentemente aprovada pelo órgão americano Food and Drugs Administration ${ }^{(30)}$ e tem demonstrado excelente atividade in vitro contra bactérias gram-positivas $^{(31)}$. Ambos foram ativos contra $100 \%$ dos estafilococos avaliados nesse estudo (Tabela 3).

O Acinetobacter spp. foi o terceiro patógeno mais freqüente. O Acinetobacter baumannii tem-se tornado um patógeno de grande importância devido a vários fatores, incluindo grande aumento de sua freqüência como causa das infecções hospitalares, especialmente pneumonia, nos últimos anos; alta mortalidade relacionada a pneumonia hospitalar por este patógeno; e também ao fato de ser normalmente resistente à maioria dos antimicrobianos utilizados na prática médica ${ }^{(18,32)}$. Com base nos resulta- 
dos do nosso estudo, podemos dizer que somente os carbapenens representam opções terapêuticas adequadas para o tratamento empírico de infecções por Acinetobacter spp. nos hospitais avaliados. A tetraciclina também apresentou boa atividade in vitro $(63,2 \%$ de sensibilidade); porém, não há estudos clínicos avaliando a eficácia clínica desse antibiótico no tratamento de infecções graves por A. baumannii. Esses resultados trazem implicações importantes, pois a alta prevalência de bacilos gram-negativos não fermentadores ( $P$. aeruginosa e Acinetobacter spp.) associadas às altas taxas de resistência desses patógenos estimulará o uso de carbapenens e conseqüentemente elevará ainda mais as taxas de resistência a esses antimicrobianos no Brasil.

A percentagem de amostras de K. pneumoniae produtoras de ESBL foi extremamente alta em nosso estudo (36\%). ESBL são $\beta$-lactamases plasmidiais, não induziveis, muito potentes. Essas enzimas degradam praticamente todos os $\beta$-lactâmicos, com exceção apenas dos carbapenens e das cefamicinas (cefoxitina). Outra característica importante dessas enzimas é que a resistência produzida por elas pode não ser detectada pelos testes de sensibilidade realizados rotineiramente pelos laboratórios de microbiologia. Isto é, apesar de cepas produtoras dessas enzimas poderem ser categorizadas como "sensiveis" a algumas cefalosporinas, poderá ocorrer falha terapêutica quando estas são utilizadas no tratamento de infecções graves $^{(14)}$. Como os testes laboratoriais podem não detectar esse tipo de resistência, recomenda-se aos laboratórios, e mesmo aos clínicos, que considerem como resistentes a todas as cefalosporinas e monobactâmicos (aztreonam) todas as amostras de K. pneumoniae e E. coli que apresentarem resistência $(\mathrm{R})$ ou resistência intermediária (I) à ceftadizima ou ceftriaxona ou cefotaxima ou aztreonam, independente do resultado do teste de sensibilidade in vitro. É importante ressaltar que, apesar de o NCCLS recomendar que as bactérias produtoras de ESBL sejam consideradas resistentes a todas as cefalosporinas, não há relatos de avaliações clínicas com cefalosporinas de quarta geração.

A cefoxitina foi ativa contra somente $80,0 \%$ das amostras de Klebsiella spp., indicando o envolvimento também de $\beta$-lactamases cromossômicas induziveis (Classe C) ou diminuição de permeabilidade, uma vez que esse composto não é degradado por ESBLs. Apesar de as ESBLs serem inibidas por inibidores de $\beta$-lactamases (ácido clavulânico, sulbactam e tazobactam), somente $70,0 \%$ das amostras de Klebsiella spp. foram sensiveis a piperacilina/tazobactam, indicando, como já demonstrado em outros estudos ${ }^{(33,34)}$, que esse composto não apresenta boa atividade in vitro contra amostras produtoras de ESBL. Outros antibióticos que apresentaram boa atividade contra Klebsiella spp. foram amicacina (MIC90, $32 \mu \mathrm{g} / \mathrm{mL}$;
$82,0 \%$ de sensibilidade) $e$ as quinolonas $(92,0 \%$ de sensibilidade).

Estudos de tipagem molecular realizados com amostras produtoras de ESBL e não apresentados mostraram grande variedade genética entre amostras produtoras de ESBL, demonstrando assim que a seleção de subpopulações mutantes em cada paciente pela exposição a antibióticos $\beta$-lactâmicos pode representar um importante fator na disseminação desse tipo de resistência no Brasil. Além disso, a possibilidade de disseminação de clones resistentes deve ser investigada em cada uma das instituições que participaram desse estudo(16).

Outra enterobactéria que se tem tornado cada vez mais freqüente e resistente a antimicrobianos é o Enterobacter spp. As taxas de resistência desse patógenos aos $\beta$ lactâmicos foi muito importante, restando apenas os carbapenens e a cefalosporina de quarta geração cefepima como $\beta$-lactâmicos que poderiam ser utilizados em terapêutica empírica quando se suspeitar de infecções causadas por esse patógeno. Além disso, esses $\beta$-lactâmicos (carbapenens e cefepima) foram ativos contra $100 \%$ das amostras de Serratia spp. avaliadas. O mecanismo de resistência responsável pela perda da atividade e do espectro de ação das cefalosporinas de terceira geração, monobactans e penicilinas de amplo espectro nessas espécies de enterobactérias é decorrente da produção de $\beta$ lactamases cromossômicas induzives, chamadas de Classe 1 ou AmpC (35,36). Espécies bacterianas, como Citrobacter, Enterobacter, Serratia e Providencia (conhecidas como grupo CESP), podem ser induzidas a produzir grandes quantidades de enzima, tornando-se assim resistentes às cefalosporinas de segunda e terceira gerações ${ }^{(35-}$ 37). O que acontece com essas espécies é que elas possuem o gene que codifica para a produção de grandes quantidades. Porém, esse gene se encontra normalmente "bloqueado". A exposição da bactéria a uma substância indutora "desbloqueia" o gene e a bactéria passa a produzir grandes quantidades. Essa característica é intrínseca de certas espécies bacterianas, como as enterobactérias do grupo CESP e a $P$. aeruginosa. Isso significa que, sempre que essas espécies forem expostas aos $\beta$-lactâmicos indutores, elas passarão a produzir grandes quantidades de $\beta$-lactamases da Classe $1^{(36-38)}$. As substâncias indutoras são os próprios $\beta$-lactâmicos, porém a capacidade de indução varia muito entre os $\beta$-lactâmicos. Os indutores mais potentes são os carbapenens e as cefamicinas (cefoxitina), seguidos pelas cefalosporinas de terceira geração(38). A importância prática desse fenômeno é que essas espécies bacterianas apresentam grande chance de desenvolver resistência a esse grupo de $\beta$-lactâmicos (cefalosporinas de terceira geração, monobactans e penicilinas de amplo espectro) durante o tratamento, com conseqüente falha terapêutica, especialmente em infecções 
com alto inóculo bacteriano, como abscessos, pneumonias e sepse, por exemplo. Dessa maneira, o uso desses $\beta$-lactâmicos no tratamento de infecções causadas por esses patógenos deve ser evitado, mesmo que a bactéria apresente sensibilidade in vitro ${ }^{(39,40)}$. Além de ser bastante estável a hidrólise por essas enzimas cromossômicas induziveis, a cefepima apresenta maior potência contra cocos gram-positivos e excelente atividade contra $P$. aeruginosa, representando assim uma importante opção para o tratamento de infecções hospitalares, especialmente infecções do trato respiratório ${ }^{(7,10,16,17,34)}$.

Em resumo, o Programa SENTRY de Vigilância de Resistência detectou alta prevalência de Acinetobacter spp. e baixa prevalência de isolamento de $S$. pneumoniae em espécimes de trato respiratório baixo de pacientes com pneumonia hospitalar internados em hospitais brasileiros. Além disso, observamos altas taxas de resistência, sendo que os principais problemas detectados foram: a) resistência a carbapenens e outros $\beta$-lactâmicos de amplo espectro em amostras de Acinetobacter spp. e P. aeruginosa; b) resistência à oxacilina em amostras de $S$. aureus; c) E. coli e K. pneumoniae produtoras de ESBLs; d) Enterobacter spp. produtores de $\beta$-lactamases cromos-

\section{REFERÊNCIAS}

1. Vincent JL, Bihari DJ, Suter PM, Bruining HA, White J. The prevalence of nosocomial infection in intensive care units in Europe. Results of the European prevalence of infection in intensive care (EPIC) study. EPIC International Advisory Committee. JAMA 1995;274:639-644.

2. Spencer RC. Predominant pathogens found in the European prevalence of infection in intensive care study. Eur J Clin Microbiol Infect Dis $1996 ; 15: 281-284$.

3. Centers for Disease Control and Prevention. Guidelines for prevention of nosocomial pneumonia. MMWR Morb Mortal Wkly Rep 1997;46: 6-7.

4. Lynch JP, Niederman MS. Controversies in respiratory infections. Semin Respir Infect 1997;12:269-270.

5. Mandell LA, Campbell GD Jr. Nosocomial pneumonia guidelines. An international perspective. Chest 1998;113:188S-193S.

6. Torres A, Aznar R, Gatell JM, Jimenez P, Gonzales J. Incidence, risk and prognosis factors of nosocomial pneumonia in mechanically ventilated patients. Am Rev Respir Dis 1990;142:523-528.

7. Jones RN. Impact of changing pathogens and antimicrobial susceptibility patterns in the treatment of serious infections in hospitalized patients. Am J Med 1996;100(Suppl 6A):3S-12S.

8. Jones RN. Can antimicrobial activity be sustained? An appraisal of orally administered drugs used for respiratory tract infections. Diagn Microbiol Infect Dis 1997;27:21-28.

9. Schleupner CJ, Cobb DK. A study of the etiologies and treatment of nosocomial pneumonia in a community-based teaching hospital. Infect Control Hosp Epidemiol 1992;13:515-525.

10. Pfaller MA, Jones RN, Doern GV, Krugler K. the Sentry Participants Group. Bacterial pathogens isolated from patients with blood stream infection: frequencies of occurrence and antimicrobial susceptibility patterns from SENTRY Antimicrobial Surveillance Program (United States and Canada, 1997). Antimicrob Agents Chemother 1998;42: $1762-1770$. sômicas induziveis (Classe C ou Grupo 1) com resistência à ceftazidima e outras cefalosporinas de terceira geração; e) resistência a fluoroquinolonas e aminoglicosídeos em várias espécies de bactérias gram-negativas. Nossos resultados enfatizam a importância dos programas de vigilância de resistência bacteriana a antimicrobianos na orientação da terapêutica empírica, especialmente em infecções em que o diagnóstico etiológico preciso é muitas vezes difícil. Esses resultados mostram também a importância da implementação de medidas de controle de infecção e racionalização do uso de antimicrobianos para evitar, ou ao menos desacelerar, a disseminação da resistência a antimicrobianos.

\section{AgRADECIMENTOS}

O autores agradecem a Adriana O. Reis, Eliete Miranda, Celso Page, Richard Hollis, Kari Kluger e P. Winokur pela importante contribuição na realização dos testes laboratoriais e análise dos resultados, $e$ a todas as pessoas que contribuíram com a coleta das amostras e dos dados epidemiológicos em cada laboratório participante. O Programa SENTRY de Vigilância de Resistência é inteiramente patrocinado pela Bristol Myers Squibb Company.

11. Reimer LG, Carroll KC. Role of the microbiology laboratory in the diagnosis of lower respiratory tract infections. Clin Infect Dis 1998; 26:742-748.

12. National Committee for Clinical Laboratory Standards (NCCLS). Methods for dilution antimicrobial susceptibility tests for bacteria that grow aerobically. 4th ed. Wayne (PA), 1997. Approved Standard, M7-A4.

13. National Committee for Clinical Laboratory Standard. Performance standards for antimicrobial susceptibility testing; tenth informational supplement M100-S10. Wayne (PA), 2000.

14. National Committee for Clinical Laboratory Standard. Performance standards for antimicrobial susceptibility testing; eighth informational supplement M100-S8. Wayne (PA), 1998.

15. Cormican MG, Marshall SA, Jones RN. Detection of extended-spectrum $\beta$-lactamase (ESBL) - producing strains by Etest ESBL screen. $J$ Clin Microbiol 1996;34:1880-1884.

16. Sader HS, Sampaio JLM, Zoccoli C, Jones RN. Results of the SENTRY antimicrobial surveillance program results in three Brazilian medical centers for 1997. Braz J Infect Dis 1999;3:63-79.

17. Sader HS, Jones RN, Gales AC, Klugler K, Pfaller MA, Doern GV, and the SENTRY Latin America Study Group. Antimicrobial susceptibility of patterns for pathogens isolated from patients in Latin American medical centers with a diagnosis of pneumonia: Results from the SENTRY Antimicrobial Surveillance Program (1997). Diag Microbiol Infect Dis 1998;32:289-301.

18. Forster DH, Daschner FD. Acinetobacter species as nosocomial pathogens. Eur J Clin Microbiol Infect Dis 1998;17:73-77.

19. Sader HS, Mendes CF, Pignatari AC, Pfaller MA. Use of macrorestriction analysis to demonstrate interhospital spread of multiresistant Acinetobacter baumannii in São Paulo, Brazil. Clin Infect Dis 1996;23: 631-634.

20. American Thoracic Society. Brazil. Hospital-acquired pneumonia in adults: diagnosis, assessment of severity, initial antimicrobial therapy, 
and preventive strategies. Am J Respir Crit Care Med 1996;153: 17111725.

21. Yangan MB. Hospital-acquired pneumonia and its management. Crit Care Nurs 1997;20:36-43.

22. Fagon JY, Chastre J, Domart Y. Nosocomial pneumonia in patients receiving continuous mechanical ventilation: prospective analysis of 52 episodes with use of a protected specimen brush and quantitative culture techniques. Am Rev Respir Dis 1989;139:877-884.

23. Rello J, Ricart M, Ausina V, Net A, Prats G. Pneumonia due to Haemophilus influenzae among mechanically ventilated patients: incidence, outcome, and risks factors. Chest 1992;102:1562-1565.

24. Talon D, Mulin B, Rouget C, Bailly P, Thouverez M, Viel JF. Risks and routes for ventilator-associated pneumonia with Pseudomonas aeruginosa. Am J Respir Crit Care Med 1998;157:978-984.

25. Rello J, Rue M, Jubert P, Muses G, Sonora R, Valles J, Niederman MS. Survival in patients with nososcomial pneumonia: impact of the severity of illness and the etiologic agent. Crit Care Med 1997;25:18621867.

26. Troillet N, Samore MH, Carmeli Y. Imipenem-resistant Pseudomonas aeruginosa: risk factors and antibiotic susceptibility patterns. Clin Infect Dis 1997;25:1094-1098.

27. Bush K, Jacoby GA, Medeiros AA. A functional classification scheme for $\beta$-lactamases and its correlation with molecular structure. Antimicrob Agents Chemother 1995;39:1211-1233.

28. Jones RN, Beach ML, Pfaller MA, Doern GV. Antimicrobial activity of gatifloxacin tested against 1676 strains of ciprofloxacin-resistant grampositive cocci isolated from patient infections in North and South America. Diag Microbiol Infect Dis 1998;32:247-252.

29. Cereda RF, Mendes CMF, Mimica L, et al. Antimicrobial activity of quinupristin/dalfopristin tested against gram-positive cocci from Brazil: Results from the Global SMART (GSMART) surveillance study. In the abstract book of the $9^{\text {th }}$ International Congress for Infectious Diseases, 2000 April 10-13; Buenos Aires, 2000.
30. Food and Drug Administration. [online] [cited 2000 sep 5] Available from: URL:http://www.fda.gov/cder/da/da.htm.

31. Rybak MJ, Hershberger E, Moldovan T, Grucz RG. In vitro activities of daptomycin, vancomycin, linezolid, and quinupristin-dalfopristin against staphylococci and enterococci, including vancomycin-intermediate and -resistant strains. Antimicrob Agents Chemother 2000;44:1062-1066.

32. Baraibar J, Correa H, Mariscal D, Gallego M, Valles J, Rello J. Risk factors for infection by Acinetobacter baumannii in intubated patients with nosocomial pneumonia. Chest 1997;112:1050-1054.

33. Gales AC, Bolmströn A, Sampaio J, Jones RN, Sader HS. Antimicrobial susceptibility of Klebsiella pneumoniae producing extended-spectrum $\beta$-lactamases (ESBL) isolated in Brazilian hospitals. Braz J Infect Dis 1997;1:196-203.

34. Sader HS, Mimiça I, Rossi F, et al. Evaluation of the in vitro activity of cefepime compared to other broad-spectrum cephalosporins against clinical isolates from 18 Brazilian hospitals by using the E test. Diagn Microbiol Infect Dis 1997;28:87-89.

35. Bush K, Jacoby GA, Medeiros AA. A functional classification scheme for $\beta$-lactamases and its correlation with molecular structure. Antimicrob Agents Chemother 1995;39:1211-1233.

36. Sanders WE, Sanders CC. Enterobacter sp.: Pathogens poised to flourish at the turn of the century. Clin Microbiol Rev 1997;10:220-241.

37. Fung-Tomc JC, Gradelski E, Huczko E, et al. Differences in the resistance variants of Enterobacter cloacae selected by extended spectrum cephalosporins. Antimicrob Agents Chemother 1996;40:1289-1293.

38. Jones RN. Important and emerging $\beta$-lactamases-mediated resistance in hospital-based pathogens: the ampC enzymes. Diagn Microbiol Infect Dis 1998;31:461-466

39. Medeiros AA. Editorial response: Relapsing infection due to Enterobacter species: Lessons of heterogeneity. Clin Infect Dis 1997;25: 341-342.

40. Quinn JP, DiVincenzo CA, Foster J. Emergence of resistance to ceftazidime during therapy for Enterobacter cloacae infections. J Infect Dis 1987; 155:942-947. 